\title{
Expanding the genetic and phenotypic spectrum of branched-chain amino acid transferase 2 deficiency
}

\section{Ina Knerr ${ }^{1}$ (D) | Roberto Colombo $^{2,3,4}$ | Jill Urquhart ${ }^{5}$ | Ana Morais ${ }^{6}$ | \\ Begona Merinero $^{6}$ | Alfonso Oyarzabal ${ }^{6}$ | Belén Pérez ${ }^{6}$ | Simon A. Jones ${ }^{5,7}$ | \\ Rahat Perveen $^{7}$ । Mary A. Preece ${ }^{8}$ | Yvonne Rogers ${ }^{1}$ | Eileen P. Treacy ${ }^{1,9}$ ।}

Philip Mayne $^{10}$ | Giuseppe Zampino ${ }^{11}$ | Sabrina MacKinnon ${ }^{12}$ | Evangeline Wassmer ${ }^{8}$ ।

Wyatt W. Yue $^{12}$ | Ian Robinson ${ }^{13}$ | Pilar Rodríguez-Pombo ${ }^{6}$ | Simon E. Olpin ${ }^{14}$ |

Siddharth Banka ${ }^{5,7}$

\footnotetext{
${ }^{1}$ National Centre for Inherited Metabolic Disorders, Temple Street Children's University Hospital, Dublin, Ireland

${ }^{2}$ Institute of Clinical Biochemistry, Faculty of Medicine, Catholic University of the Sacred Heart, Rome, Italy

${ }^{3}$ Policlinico Agostino Gemelli, Rome, Italy

${ }^{4}$ Center for the Study of Rare Hereditary Diseases, Niguarda Ca' Granda Metropolitan Hospital, Milan, Italy

${ }^{5}$ Manchester Centre for Genomic Medicine, St Mary's Hospital, Manchester University NHS Foundation Trust, Health Innovation Manchester,

Manchester, UK

${ }^{6}$ Centro de Diagnostico de Enfermedades Moleculares, Departamento de Biologia Molecular, Centro de Biologia Molecular Severo Ochoa, Centro de Investigacion Biomedica en Red de Enfermedades Raras, Universidad Autonoma de Madrid, Spain

${ }^{7}$ Division of Evolution and Genomic Sciences, School of Biological Sciences, Faculty of Biology, Medicine and Health, University of Manchester, Manchester, UK

${ }^{8}$ Children's Hospital, Birmingham Women's and Children's NHS Foundation Trust, Birmingham, UK

${ }^{9}$ Adult Metabolic Service, Mater Misericordiae University Hospital, Dublin, Ireland

${ }^{10}$ Department of Biochemistry, Temple Street Children's University Hospital, Dublin, Ireland

${ }^{11}$ Department of Paediatrics, Catholic University of the Sacred Heart, and Center for Rare Diseases, Policlinico Agostino Gemelli, Rome, Italy

${ }^{12}$ Structural Genomics Consortium, Nuffield Department of Medicine, University of Oxford, Oxford, UK

${ }^{13}$ Department of Radiology, Temple Street Children's University Hospital, Dublin, Ireland

${ }^{14}$ Department of Clinical Chemistry, Sheffield Children's NHS Foundation Trust, Sheffield Children's Hospital, Sheffield, UK
}

\section{Correspondence}

Ina Knerr, UCD School of Medicine, National Centre for Inherited Metabolic Disorders, Temple Street Children's

University Hospital, Dublin 1, Ireland.

Email: ina.knerr@cuh.ie

Funding information

Fundación Isabel Gemio; MINECO-FEDER;

Fondo Europeo de Desarrollo Regional,

Grant/Award Number: PI12/02078;

Ministerio de Economía y Competitividad

Communicating Editor: Bridget Wilcken

\section{Abstract}

The first step in branched-chain amino acid (BCAA) catabolism is catalyzed by the two BCAA transferase isoenzymes, cytoplasmic branched-chain amino acid transferase (BCAT) 1, and mitochondrial BCAT2. Defects in the second step of BCAA catabolism cause maple syrup urine disease (MSUD), a condition which has been far more extensively investigated. Here, we studied the consequences of BCAT2 deficiency, an ultra-rare condition in humans. We present genetic, clinical, and functional data in five individuals from four different families with homozygous or compound heterozygous BCAT2 mutations which were all detected following abnormal biochemical profile results or familial mutation segregation studies. We demonstrate that BCAT2 deficiency has a recognizable biochemical profile with raised plasma BCAAs and, in contrast with MSUD, low-normal branched-chain 
keto acids (BCKAs) with undetectable L-allo-isoleucine. Interestingly, unlike in MSUD, none of the individuals with BCAT2 deficiency developed acute encephalopathy even with exceptionally high BCAA levels. We observed wide-ranging clinical phenotypes in individuals with BCAT2 deficiency. While one adult was apparently asymptomatic, three individuals had presented with developmental delay and autistic features. We show that the biochemical characteristics of BCAT2 deficiency may be amenable to protein-restricted diet and that early treatment may improve outcome in affected individuals. BCAT2 deficiency is an inborn error of BCAA catabolism. At present, it is unclear whether developmental delay and autism are parts of the variable phenotypic spectrum of this condition or coincidental. Further studies will be required to explore this.

\section{K E Y W O R D S}

autism spectrum disorder, BCAT2, branched-chain amino acids, branched-chain amino transferase 2, encephalopathy, maple syrup urine disease

\section{1 | INTRODUCTION}

There are three proteinogenic branched-chain amino acids (BCAAs), leucine, isoleucine, and valine. The first enzymatic step in BCAA metabolism is carried out by branched-chain amino acid transferase (BCAT), a pyridoxine-dependent enzyme which utilizes $\alpha$-ketoglutarate to form branchedchain $\alpha$-keto acids (BCKAs) (Figure 1A). ${ }^{1}$ Expression of BCATs is mainly extra-hepatic and is highest in the skeletal muscle with some expression in adipose tissue, kidneys, intestine, heart, and the brain. ${ }^{2}$ BCATs exist in two isoforms: cytosolic (BCAT1) encoded by BCAT1 (OMIM 113520), and mitochondrial (BCAT2) encoded by BCAT2 (OMIM 113530). These isoforms share $58 \%$ homology, but vary in cellular location, tissue distribution, and catalytic efficiency. Mitochondrial BCAT2 is the more ubiquitous of the two isoforms. ${ }^{3}$ In humans, immunoreactivity for BCAT2 has been shown to be present throughout the brain with consistent labeling of vascular endothelial cells in the grey and white matter, whereas the cytosolic BCAT1 is restricted to neurons. ${ }^{3}$ Thus far, there has been only one genetically confirmed published case of BCAT2 deficiency in a 25-year-old man with headaches and mild memory impairment without developmental delay. ${ }^{4}$

In the liver, BCKAs undergo irreversible oxidative decarboxylation by BCKA-dehydrogenase complex (BCKDH) and its cofactor thiamine (Figure 1A). ${ }^{5}$ Maple syrup urine disease (MSUD, OMIM 248600) is a well-known disorder of BCAA metabolism ${ }^{6}$; it is caused by defects in the BCKADH complex mediated second step in the catabolism of $\mathrm{BCAAs}^{7-9}$ (Figure 1A). The biochemical hallmarks of MSUD are grossly elevated concentrations of BCAAs, especially leucine, and their corresponding BCKAs and hydroxy-acids together with abnormal quantities for L-alloisoleucine. ${ }^{10}$ Untreated neonates with classical MSUD develop life-threatening metabolic crisis with encephalopathy but variant forms of MSUD with residual BCKDH activity may present later in life, for example, with developmental delay, intermittent vomiting, or failure to thrive. In affected individuals, metabolic stress due to intercurrent infections or prolonged fasting can precipitate severe endogenous intoxication with acute encephalopathy and also peripheral neuropathy. ${ }^{11}$ The chronic phenotype of MSUD is largely attributed to the neurotoxicity of leucine resulting in deficiency of several critical amino acids in the brain. ${ }^{12}$ The biochemical basis of acute toxicity along with encephalopathy in MSUD is incompletely understood. It has been shown in an animal model that chronic administration of BCAAs at millimolar concentrations can cause significant impairment in memory tasks in rats along with an increase in brain-derived neurotrophic factor (BDNF) in the hippocampus and cerebral cortex, and that oxidative stress may contribute BCAA-induced neurological impairment. ${ }^{13}$ However, the acute exposure of brains of young rats to the toxic BCKA $\alpha$-ketoisocaproic acid led to a decrease in BDNF as well as nerve growth factor concentrations. ${ }^{14}$

Here, we describe the biochemical and clinical features of five individuals with bi-allelic $B C A T 2$ variants to establish the distinct metabolic profile of BCAT2 deficiency and highlight the uncertainties of its clinical presentation.

\section{2 | METHODS AND RESULTS}

The study was approved by the local ethics committees (UK NHS ethics committee 11/H1003/3; CEI-28-701 for ethics committee of the Universidad Autónoma de Madrid, 


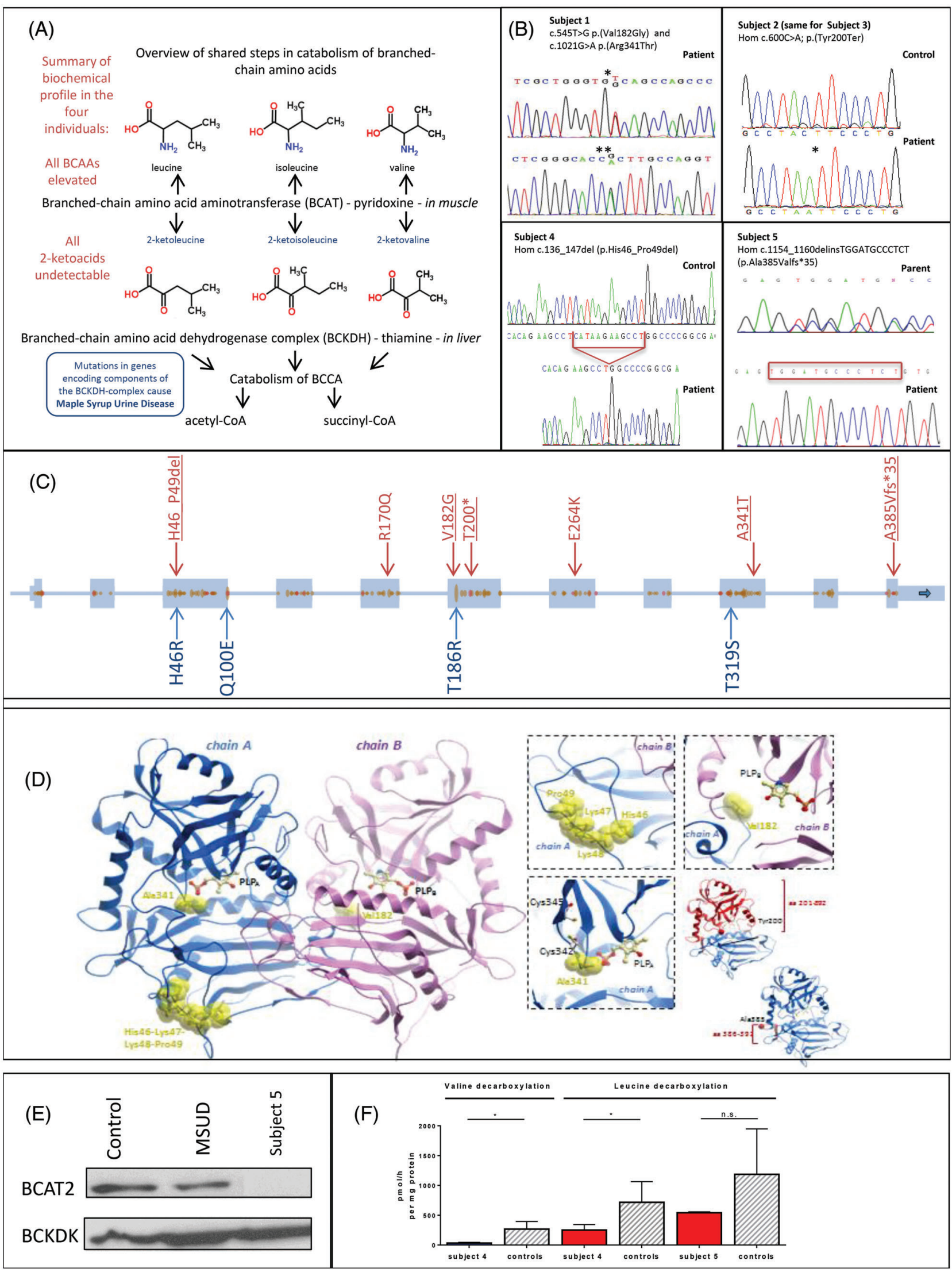

FIG URE 1 Legend on next page. 
Spain, TSCUH research and ethics committee 12.043). All procedures followed were in accordance with the ethical standards of the responsible committee on human experimentation (institutional and national) and with the Helsinki Declaration of 1975, as revised in 2000. Genetic testing was performed according to Institutional Review Board (IRB) approved guidelines or as part of clinical service with appropriate informed consent. We evaluated patients' characteristics and compared available clinical, biochemical, and molecular genetic findings.

Subject 1 is a 29 -year-old Italian man with moderate global developmental delay and autism spectrum disorder. His full scale, verbal, and performance intelligence quotient (Wechsler Adult Intelligence Scale, WAIS-R) scores at the age of 28 years were 63, 67, and 59, respectively. We performed trio (proband and both parents) whole exome sequencing (WES) and identified a paternally inherited BCAT2 c.545T $>\mathrm{G}$ (p.Val182Gly) and a maternally inherited BCAT2 c.1021G >A (p.Ala341Thr) variant in the proband (nomenclature according to the longest transcript NM 001190, ENST00000316273.10) (Supplementary Methods). No other likely causal pathogenic variants were identified. Both $B C A T 2$ variants were confirmed by Sanger sequencing using standard methods (Figure 1B). Biochemical phenotyping $^{15}$ revealed elevated valine, leucine, and isoleucine concentrations in plasma (Table 1) (Supplementary Methods).

Subject 2, an 11-year-old girl, was one of two children born to consanguineous parents of Pakistani ancestry and presented at the age of 2 years with global developmental delay and microcephaly. She was diagnosed with learning difficulties and ataxia along with signs of spastic paraparesis. Her MRI brain at 8 years of age showed that the corpus callosum was broad and globular in appearance and also some mild cerebral T2 white matter hyperintensities posteriorly (Figure 2). The proband has a younger brother with global developmental delay and motor impairment. He was detected to have a homozygous c.263dupG, p. (Leu89fsTer13) variant in the exon 3 of B4GALNT1 via a multigene next generation sequencing (NGS) panel. Biallelic B4GALNT1 variants cause hereditary spastic paraplegia type 26 (OMIM \# 609195, ${ }^{16}$ ). Testing in the proband revealed that she too was homozygous for the frameshift B4GALNT1 mutation. However, her initial biochemical investigations also revealed elevated plasma valine, leucine, and isoleucine concentrations (Table 1, Supplementary Methods), organic acids including BCKAs were normal (data not shown). She did not have any history of acute deterioration or encephalopathy. We performed targeted BCAT2 Sanger sequencing and identified a novel homozygous nonsense $B C A T 2$ c.600C $>$ A (p.Tyr200Ter) variant (Figure 1B).

F I G URE 1 Catabolism of branched-chain amino acids (BCAAs) and functional consequences of BCAT2 mutations. (A) Overview of shared steps in catabolism of BCAAs and the biochemical profile of BCAT2 deficiency. In muscles, leucine, isoleucine, and valine undergo reversible transamination to their respective branched-chain keto acids (BCKAs) by BCAA aminotransferase (BCAT). In liver, BCKAs undergo oxidative decarboxylation catalyzed by BCKA-dehydrogenase complex (BCKDH). Subsequent degradation pathways of BCAA diverge. Maple syrup urine disease is caused by BCKDH deficiency leading to grossly elevated BCAAs and their corresponding BCKAs and hydroxy-acids. In BCAT2 deficiency, BCAAs are excessively raised in plasma but the corresponding BCKAs were undetectable or low-normal (the biochemical profile is provided in red font on the left side). (B) Chromatograms from Sanger sequencing. Parts of chromatograms of BCAT2 Sanger sequencing showing compound heterozygous c.545T $>$ G p.(Val182Gly) (marked by *) and c.1021G $>$ A (p.Ala341Thr) (marked by **) mutations in subject 1; homozygous nonsense c.600C >A (p.Tyr200Ter) in subject 2 (similar to subject 3); homozygous in-frame BCAT2 c.136_147 del; (p. His46_Pro49del) in subject 4; and homozygous c.1154_1160delinsTGGATGCCCTCT (p.Ala385Valfs*35) in subject 5. All variants are named according to NM_001190.3. (C) Location of homozygous or compound heterozygous BCAT2 variants. Wide and narrow blue boxes represent exons and introns respectively. Brown and red ovals within exons depict missense and loss of function variants in the general population respectively. The size of the ovals is proportional to the frequency of the variant. Red arrows depict the positions of the pathogenic mutations detected in individuals with BCAT2 deficiency and font. Novel mutations reported in this study are underlined. Positions of homozygous variants in the general population (from ExAC database) are depicted using blue arrows. (D) Protein structural analysis of BCAT2 variants. Left panel: the three novel missense and deletion mutations in this study (shown as yellow sticks and spheres) are mapped onto the crystal structure of human BCAT2 (PDB code 1EKF), which arranges as a homodimer (colored blue and pink chains), with each subunit containing an active site-bound PLP cofactor (shown as sticks). Right panels: magnified views showing the local environment of the three sites of missense mutation (namely His46-Lys47-Lys48-Pro49, Val182, and Ala341), as well as cartoon representation highlighting the regions of the BCAT2 protein deleted (red) for the two nonsense mutations.

(E) BCAT2 western blot analysis. Western blot analysis of protein extracted from cellular and nuclear lysates of fibroblasts from a control subject, an individual with maple syrup urine disease (MSUD) and subject 5. BCAT2 protein was found to be completely absent in subject 5 with homozygous c.1154_1160delinsTGGATGCCCTCT (p.Ala385Valfs*35) mutation. (F) BCAT activity analysis. Decarboxylation was measured in the form of release of ${ }^{14} \mathrm{CO}_{2}$ from L- $\left[1-{ }^{14} \mathrm{C}\right]$ leucine and ${ }^{14} \mathrm{CO}_{2}$ from $\mathrm{L}-\left[1-{ }^{14} \mathrm{C}\right]$ valine. Results are expressed as pmol/mg protein/hour. Data are representative of three independent experiments and is given as mean $\pm \mathrm{SD}$ (controls are shown with a hatching pattern). Significantly reduced decarboxylation of leucine and valine was detected in fibroblasts from subject 4 (*, $P<.05$, Mann-Whitney $U$ test). Leucine decarboxylation was also measured in fibroblasts of subject 5 and that was reduced but not statistically significant (n.s.). These results support impaired BCAT activity but do also point towards some residual decarboxylation capacity, possibly at cytosolic BCAT level 


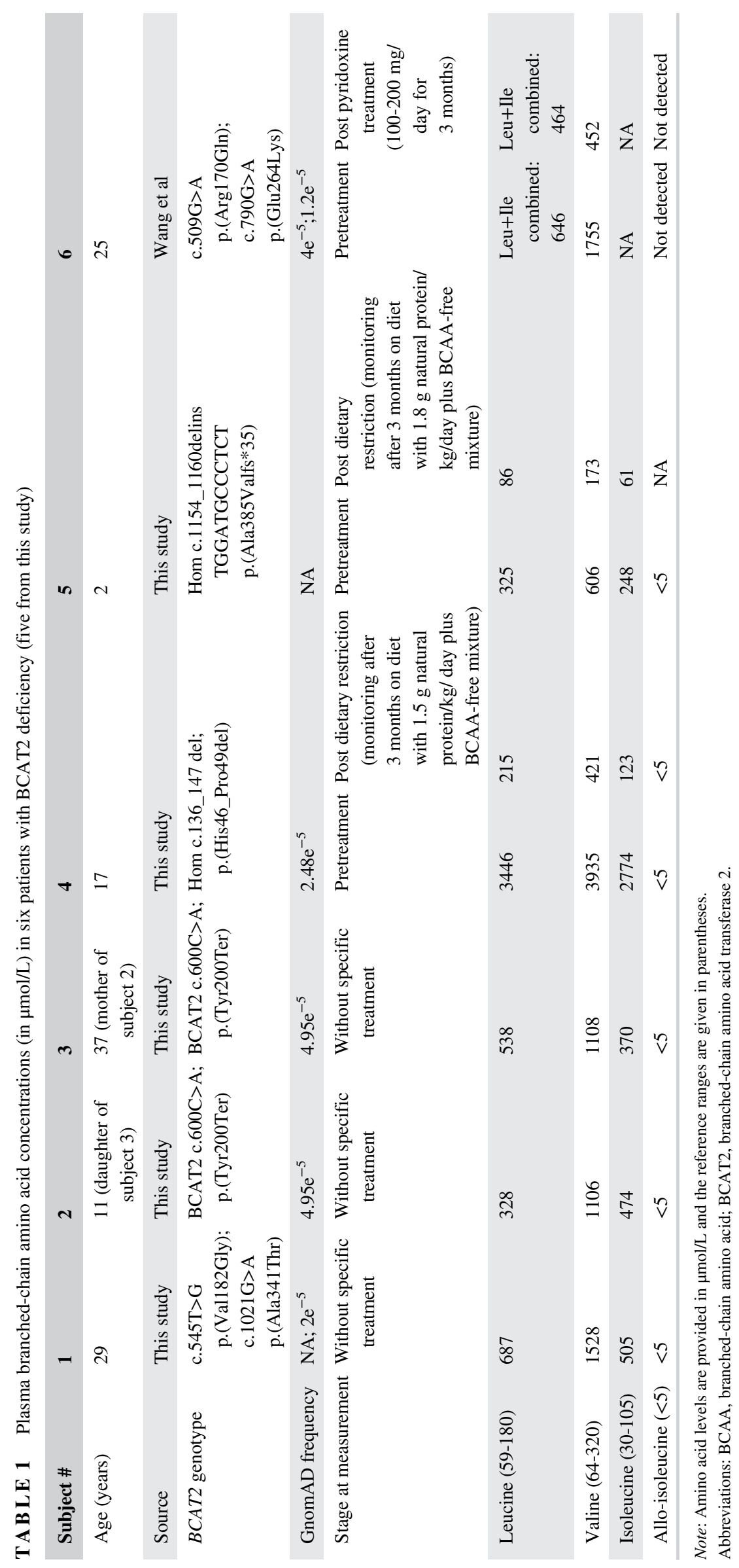



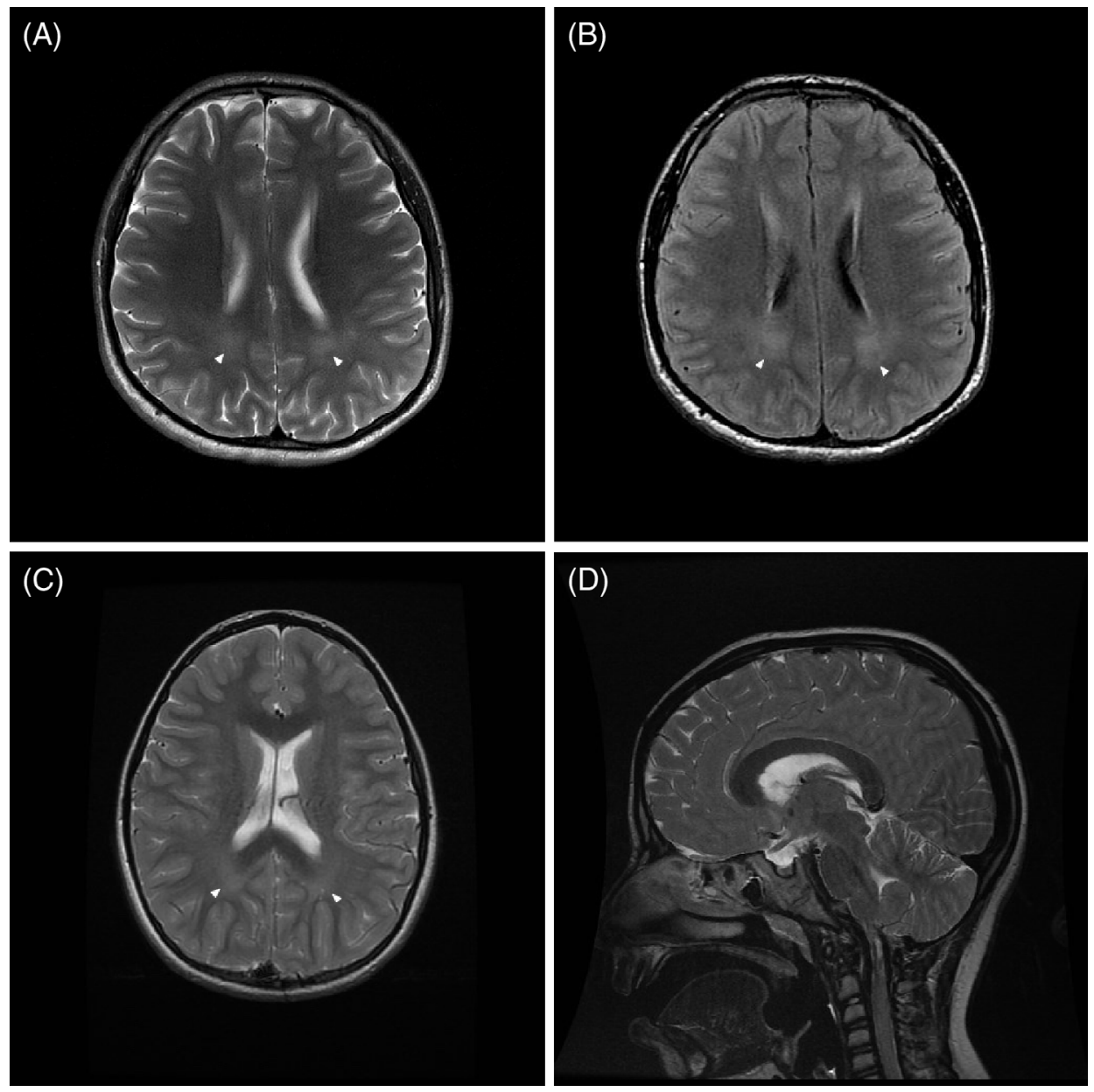

FIGURE 2 MRI brain images of subject 4 (17-year-old boy with BCAT2 deficiency) and subject 2 (11-year-old girl with BCAT2 deficiency and hereditary spastic paraplegia type 26). Axial T2 (A) and T2 FLAIR sequence (B) demonstrating mild increased $\mathrm{T} 2$ hyperintensity within the posterior periventricular white matter (white arrows) in subject 4 at 17 years of age. Mild nonspecific increased $\mathrm{T} 2$ signal $(\mathrm{C})$ in the posterior cerebral white matter for her age in subject 2 (axial T2 sequence at 8 years of age) and slightly broad and globular appearance of the corpus callosum (sagittal T2) (D). BCAT2, branchedchain amino acid transferase 2
Her clinical features did not respond to pyridoxine supplements. Her brother and father were found to be carriers for the familial BCAT2 variant.

Subject 3 is the mother of case 2; she underwent biochemical and genetic testing after her daughter was identified with BCAT2 deficiency. The family also has two unaffected children (2 years and 13 years old). The woman did not report any acute symptoms and her systemic examination was entirely normal. She did not report any previous major illnesses or complications during her four pregnancies. Proband 3 does not have intellectual disability but we did not perform any formal tests of cognition. Her baseline biochemical investigations, while she continues to be on a normal diet, showed high BCAAs with no ketones/BCKAs or allo-isoleucine detectable (Table 1).

Subject 4 is a 17-year-old boy of Irish ancestry with severe speech and language impairment, intellectual disability, and autism spectrum disorder. He had two words at 1 year of age and walked at 18 months. There was no further progression in language skills and mannerisms occurred around 2 years of age, such as hand flapping. A diagnosis of autism was made at 2 years of age. He was first referred to the metabolic department for an assessment when he was
4 years old. At the time, plasma amino acid measurements showed exceptionally high levels of BCAAs (Table 1) with undetectable L-allo-isoleucine, BCKAs, and ketones in plasma and urine. He never had an altered state of consciousness but he was admitted for further work-up in view of his high BCAA concentrations; he underwent an urgent CT brain scan which was normal. His high BCAA concentrations were confirmed in a separate blood sample (ie, leucine $2067 \mu \mathrm{M} / \mathrm{L}$, valine 4045 , isoleucine 1677 , L-alloisoleucine not detectable; for reference ranges see Table 1) before he was commenced on a protein-restricted diet with BCAA-free amino acids supplements. In terms of his adaptive functioning, he obtained an Adaptive Behavior Composite $(\mathrm{ABC})$ score of $43(<1$ percentile) on the Vineland Adaptive Behavior Scales (2nd Edition, VABS-II, 2005) at 6 years of age.

His brain MRI showed mild nonspecific increased T2 FLAIR signal within the posterior periventricular white matter with a normal parenchymal signal elsewhere (Figure 2). He also had a normal MR spectroscopy (MRS) at age 17 years (data not shown). We performed clinical targeted BCAT2 Sanger sequencing and identified a novel homozygous in-frame BCAT2 c.136_147del (p.His46_Pro49del) 
deletion (Figure 1B). This variant was confirmed to be heterozygous in the proband's father; the mother was not available for testing. While he responded biochemically well to the diet (Table 1), there was no improvement in his clinical features over the years (severe autism), and his diet was relaxed during adolescence, again with no noticeable clinical effect.

Subject 5, a Spanish girl of was identified to have slight increase of valine on expanded newborn bloodspot screening at 48 hours of age $(312 \mu \mathrm{mol} / \mathrm{L}$; normal range for newborns 54-270). On day 13 of life, further metabolic work-up was performed including plasma BCAAs (Table 1). BCKAs, in particular the leucine-derived metabolites, were normal or only marginally detectable (data not shown). The girl was commenced on a BCAA-restricted diet from 2 weeks of life (Table 1). Targeted exome sequencing revealed a homozygous frameshift c.1154_1160delinsTGGATGCCCTCT (p. Ala385Valfs*35) BCAT2 deletion which was confirmed by Sanger sequencing (Figure 1B). The girl continues to be on a moderate BCAA-restricted diet with no additional supplements with BCAA plasma concentrations close to normal (Table 1). Currently, at 2.5 years of age she is developing normally and is clinically asymptomatic.

We undertook in silico analysis of all the potentially pathogenic variants identified in this study. We here included two previously published compound heterozygous $B C A T 2$ variants (p.Arg170Gln and p.Glu264Lys) in our analysis. ${ }^{4}$ We also included all the $B C A T 2$ variants (p.His46Arg, p.Gln100Glu, p.Thr186Arg, and p.Thr319Ser) that have been reported to be present in homozygous state in the general population on the ExAC database (http://exac. broadinstitute.org/gene/ENSG00000105552) (Figure 1C). We used a pyridoxal phosphate (PLP) bound wild type human BCAT2 structure (PDB accession code: 1ekf) to examine the residue positions and visualize the site of the all nine BCAT2 variants. Structural analysis was performed with ICM Pro (Molsoft, LLC) (Figure 1D). The seven variants identified in individuals with BCAT2 deficiency were predicted to be damaging, whereas all the homozygous ExAC variants were likely to be benign (Table S1) (Figure 1D).

The mutation detected in subject 5 was present in the last exon; however, western blot analysis (polyclonal antiBCAT2 \#9432 antibody against peptide residues near the amino terminus, Cell Signaling Technology) demonstrated a total absence of BCAT2 in her fibroblasts (Figure 1E).

Oxidative decarboxylation rates for leucine and valine were determined in cultured skin fibroblasts by measuring release of ${ }^{14} \mathrm{CO}_{2}$ from $\mathrm{L}-\left[1-{ }^{14} \mathrm{C}\right]$ leucine and $\mathrm{L}-\left[1-{ }^{14} \mathrm{C}\right]$ valine, respectively, by a modification of previous methodologies (Supplementary methods). Release of ${ }^{14} \mathrm{CO}_{2}$ from L- $\left[1-{ }^{14} \mathrm{C}\right]$ leucine and from $\mathrm{L}-\left[1-{ }^{14} \mathrm{C}\right]$ valine was reduced in subject 4 's fibroblasts demonstrating reduced capacity of the cells to metabolize leucine and valine (Figure 1F). Marked reduction of $\left[1-{ }^{14} \mathrm{C}\right]$ leucine decarboxylation $(18.3 \%$ of intra-assay control value) was also detected in fibroblasts from subject 5 , confirming the pathogenicity of the homozygous frameshift variant (Figure 1F).

All individuals with pathogenic BCAT2 variants demonstrated a distinct biochemical profile with raised plasma BCAA levels (Table 1). Plasma valine concentrations in all five individuals were considerably higher compared with leucine or isoleucine. These findings are in line with our studies performed on fibroblasts of subject 4 that showed a more pronounced decrease in release of ${ }^{14} \mathrm{CO}_{2}$ from $\left[1-{ }^{14} \mathrm{C}\right]$ valine (Figure 1F). Unlike in MSUD, ${ }^{10}$ BCKAs were low or undetectable in individuals with BCAT deficiency even in the presence of high BCAA concentrations. This is consistent with the block at the BCAAs transamination level (Figure 1A). We also did not observe an increase in L-alloisoleucine in these individuals with BCAT2 deficiency, another biochemical marker differentiating this disorder from $\mathrm{MSUD}^{17}$; this is because in vivo L-allo-isoleucine is formed via retransamination of 3-methyl-2-oxopentanoate, which is not formed in BCAT2 deficiency.

\section{3 | DISCUSSION}

NGS, or high-throughput sequencing, has transformed the diagnosis of known and new rare diseases. We recently showed the complementary value of NGS in diagnosis of inborn errors of metabolism. ${ }^{18}$ In this study, a combination of NGS and targeted Sanger sequencing guided or followed by biochemical analysis leads to identification of five individuals with $B C A T 2$ mutations. Homozygous null variants were identified in subjects 2, 3, and 5. Subject 1 had compound heterozygous missense variants, whereas subject 4 had a homozygous in-frame deletion. In silico analysis of these variants supported their pathogenicity (Figure 1D) (Table S1). Furthermore, none of these variants have been described in the general population in homozygous state in ExAC and gnomAD databases (https://www.biorxiv.org/ content/10.1101/531210v2. article-info $^{19}$ ). Absence of protein was demonstrated in fibroblasts from subject 5 (Figure $1 \mathrm{E})$. We demonstrated reduced enzyme activity in two affected individuals (Figure 1F).

The biochemical phenotype of BCAT2 deficiency is distinct from MSUD and is characterized by raised plasma BCAA levels in the absence of BCKAs and L-allo-isoleucine. Remarkably, none of the individuals affected with BCAT2 deficiency have ever developed acute encephalopathy, although their plasma BCAAs were persistently elevated, in some cases close to the acute encephalopathy range of MSUD. Decreasing the plasma leucine is considered to be 
one of the primary goals of treatment of acute decompensation in MSUD. ${ }^{20}$ Our observations suggest that high levels of BCAAs are unlikely to be solely responsible for acute decompensation in MSUD and that partial blocking of BCAT2 activity may be an additional therapeutic tool in the future to treat acute episodes of MSUD.

Prior to our study, there has been only one genetically confirmed published case of BCAT2 deficiency, a 25-yearold man (individual 6 in Table 1) with headaches and mild memory impairment but no developmental delay. ${ }^{4}$ The clinical findings of five additional individuals presented here raise important questions about the clinical phenotype of BCAT2 deficiency. It remains unclear at present if BCAT2 deficiency could present as an adult onset disorder (as in individual 6) and the findings reported in younger subjects may not reflect its true phenotype, comparable to, for example, 3-methylglutaconic aciduria type 1, a rare inborn error of leucine catabolism. ${ }^{21}$ However, none of the subjects presented here, which includes two adults, have a history of headaches and mild memory impairment, and it is possible that these two clinical features affect only some individuals with BCAT2 deficiency. Furthermore, three of five individuals presented had developmental delay and/or autistic features. Notably, no other cause of developmental problems was detected in subject 1 who underwent WES.

In subject 2 who was born to consanguineous parents, two separate autosomal recessive conditions could be identified along with a distinctive neurological phenotype which is largely attributed to hereditary spastic paraplegia. Of note, both conditions have different cytogenetic locations (19q13.33 for BCAT2 and 12q13.3 for spastic paraplegia 26). As part of family studies we detected one affected individual (subject 3) with no obvious phenotype. However, two members of the same family were included in this case series whereby one of them had a compounded disorder which may contribute to the phenotypic variability of BCAT2 deficiency described.

In most cases the genetic diagnosis of BCAT2 deficiency was prompted by the biochemical analysis. Amino acid profiling is usually performed in individuals with developmental problems. Hence, our detection of BCAT2 deficiency in patients with developmental problems may be due to ascertainment bias. However, we could demonstrate abnormal brain MRI findings in subject 4 for which no other underlying cause except BCAT2 deficiency could be identified.

Notably, BCAAs are important nitrogen donors for glutamate, the major excitatory neurotransmitter, and $\gamma$-amino butyric acid (GABA), a widely distributed inhibitory neurotransmitter in the brain. ${ }^{22,23}$ The coordinated activities of BCAT1, BCAT2, as well as BCKDH are crucial for maintaining the balance of glutamate and GABA in the brain. ${ }^{22}$ Dysregulation of both the neurotransmitters has been implicated in pathogenesis of intellectual disability and autism. ${ }^{24}$
A protein-restricted diet in subject 4 after diagnosis showed a dramatic reduction in the levels of BCAAs with an overall static clinical presentation. Subject 5 (who has a null mutation) was started on protein-restricted diet soon after birth and she has not shown any signs of developmental delay. These observations suggest that the biochemical markers of BCAT2 deficiency may respond to dietary management. Importantly, the clinical course of subject 5 suggests that early treatment of BCAT2 deficiency may be crucial. However, larger cohorts of individuals with BCAT2 deficiency need to be studied to investigate the impact of dietary management on the clinical aspects of this condition.

Interestingly, some individuals with MSUD are thought to be responsive to thiamine, the cofactor for BCKDH. ${ }^{25}$ Similarly, pyridoxine supplementation in BCAT2 deficiency may be be a potential treatment in individuals with missense mutations where the cofactor may help to stabilize the enzyme e. ${ }^{4}$ Subject 2 in our study, with a premature termination of the BCAT2 protein, did not respond to pyridoxine treatment. Subject 4 was also commenced on a trial of pyridoxine $(100 \mathrm{mg} /$ day $)$ at 16 years of age with no clinical improvement (in addition to his protein-restricted diet which was started when he was 4 years old). Of note, the in-frame $B C A T 2$ deletion in this individual is distant from the PLP binding site.

Collectively, our study demonstrates that BCAT2 deficiency is an inborn error of BCAA catabolism that results in a distinctive biochemical profile with raised plasma BCAA levels in the absence of BCKAs and L-allo-isoleucine. BCAT2 deficiency may be associated with developmental delay and autism spectrum disorder in some cases; however, its phenotype appears to be extremely variable and further studies are needed to explore the clinical consequences of BCAT2 deficiency in more detail. Timely diagnosis and treatment may potentially improve the outcome in clinically affected patients. Taken together, our observations provide further insight into catabolism of BCAAs and their genetic defects in humans.

\section{ACKNOWLEDGMENTS}

The authors are indebted to all patients and their families for their assistance and support. We express our thanks to all those involved in patient care. This study was supported in part by Spanish Ministerio de Economía y Competitividad and Fondo Europeo de Desarrollo Regional (FEDER) (PI12/02078), MINECO-FEDER (to P.R.P.) and Fundación Isabel Gemio (to B.P.).

\section{CONFLICT OF INTEREST}

The authors declare no potential conflict of interest. 


\section{ORCID}

Ina Knerr $(10$ https://orcid.org/0000-0002-4015-0658

\section{REFERENCES}

1. Hall TR, Wallin R, Reinhart GD, Hutson SM. Branched chain aminotransferase isoenzymes purification and characterization of the rat brain isoenzymes. J Biol Chem. 1993;268:30923098.

2. Brosnan JT, Brosnan ME. Branched-chain amino acids: enzyme and substrate regulation. $J$ Nutr. 2006;136:207S-211S.

3. Hull J, Hindy ME, Kehoe PG, Chalmers K, Love S, Conway ME. Distribution of the branched chain aminotransferase proteins in the human brain and their role in glutamate regulation. J Neurochem. 2012;123:997-1009.

4. Wang XL, Li CJ, Xing Y, Yang YH, Jia JP. Hypervalinemia and hyperleucine-isoleucinemia caused by mutations in the branchedchain-amino-acid aminotransferase gene. J Inherit Metab Dis. 2015;38:855-861.

5. Koike M, Koike K, Hiraoka T. Structure, function and an inherited defect of 2-oxo acid dehydrogenase multienzyme complexes. Biochem Soc Trans. 1981;9:6-7.

6. De Baulny HO, Dionisi-Vici C, Wendel U. Branched-chain organic acidurias/acidaemias. In: Saudubray JM, Van den Berghe G, Walter JH, eds. Inborn Metabolic Diseases. Berlin, Heidelberg: Springer; 2012:277-296.

7. Herring WJ, Litwer S, Weber JL, Danner DJ. Molecular genetic basis of maple syrup urine disease in a family with two defective alleles for branched chain acyltransferase and localization of the gene to human chromosome 1. Am J Hum Genet. 1991;48: 342-350.

8. Nobukuni Y, Mitsubuchi H, Akaboshi I, et al. Maple syrup urine disease complete defect of the E1 beta subunit of the branched chain alpha-ketoacid dehydrogenase complex due to a deletion of an 11-bp repeat sequence which encodes a mitochondrial targeting leader peptide in a family with the disease. J Clin Invest. 1991;87: 1862-1866.

9. Zhang B, Edenberg HJ, Crabb DW, Harris RA. Evidence for both a regulatory mutation and a structural mutation in a family with maple syrup urine disease. J Clin Invest. 1989;83:1425-1429.

10. Schadewaldt P, Bodner-Leidecker A, Hammen HW, Wendel U. Significance of L-alloisoleucine in plasma for diagnosis of maple syrup urine disease. Clin Chem. 1999;45:1734-1740.

11. Harty S, King MD, McCoy B, Costigan D, Treacy EP. Sensorymotor polyneuropathy occurring in variant maple syrup urine disease. J Inherit Metab Dis. 2008;31:209-211.

12. Araújo P, Wassermann GF, Tallini K, et al. Reduction of large neutral amino acid levels in plasma and brain of hyperleucinemic rats. Neurochem Int. 2001;38:529-537.

13. Scaini G, Comim CM, Oliveira GM, et al. Chronic administration of branched-chain amino acids impairs spatial memory and increases brain-derived neurotrophic factor in a rat model. J Inherit Metab Dis. 2013;36:721-730.
14. Wisniewski MS, Carvalho-Silva M, Gomes LM, et al. Intracerebroventricular administration of $\alpha$-ketoisocaproic acid decreases brain-derived neurotrophic factor and nerve growth factor levels in brain of young rats. Metab Brain Dis. 2016;31: 377-383.

15. De Goede C, Yue WW, Yan G, et al. Role of reverse phenotyping in interpretation of next generation sequencing data and a review of INPP5E related disorders. Eur J Paediatr Neurol. 2016;20:286-295.

16. Boukhris A, Schule R, Loureiro JL, et al. Alteration of ganglioside biosynthesis responsible for complex hereditary spastic paraplegia. Am J Hum Genet. 2013;93:118-123.

17. Schadewaldt P, Bodner-Leidecker A, Hammen HW, Wendel U. Formation of L-alloisoleucine in vivo : an $\mathrm{L}-\left[{ }^{13} \mathrm{C}\right]$ isoleucine study in man. Pediatr Res. 2000;47:271-277.

18. Ghosh A, Schlecht H, Heptinstall LE, et al. Diagnosing childhoodonset inborn errors of metabolism by next-generation sequencing. Arch Dis Child. 2017;102:1019-1029.

19. Karczewski KJ, Francioli LC, Tiao G, et al. Variation across 141,456 human exomes and genomes reveals the spectrum of lossof-function intolerance across human protein-coding genes. Biorxiv. 2019; https://www.biorxiv.org/content/10.1101/531210v2.

20. Strauss KA, Puffenberger EG, Morton DH. Maple syrup urine disease. In: Pagon RA, Adam MP, Ardinger HH, et al., eds. GeneReviews. Seattle, WA: University of Washington, Seattle; 2013.

21. Wortmann SB, Kremer BH, Graham A, et al. 3-Methylglutaconic aciduria type I redefined: a syndrome with late-onset leukoencephalopathy. Neurology. 2010;75:1079-1083.

22. Cole JT, Sweatt AJ, Hutson SM. Expression of mitochondrial branched-chain aminotransferase and $\alpha$-keto-acid dehydrogenase in rat brain: implications for neurotransmitter metabolism. Front Neuroanat. 2012;6:18.

23. Hutson SM, Islam MM, Zaganas I. Interaction between glutamate dehydrogenase (GDH) and L-leucine catabolic enzymes: intersecting metabolic pathways. Neurochem Int. 2011;59:518-524.

24. De la Torre-Ubieta L, Won H, Stein JL, Geschwind DH. Advancing the understanding of autism disease mechanisms through genetics. Nat Med. 2016;22:345-361.

25. Chuang JL, Wynn RM, Moss CC, et al. Structural and biochemical basis for novel mutations in homozygous Israeli maple syrup urine disease patients. J Biol Chem. 2004;279:17792-17800.

\section{SUPPORTING INFORMATION}

Additional supporting information may be found online in the Supporting Information section at the end of this article.

How to cite this article: Knerr I, Colombo R, Urquhart $\mathrm{J}$, et al. Expanding the genetic and phenotypic spectrum of branched-chain amino acid transferase 2 deficiency. J Inherit Metab Dis. 2019; 42:809-817. https://doi.org/10.1002/jimd.12135 